\title{
AVALIAÇÃO DA FORÇA MUSCULAR EM PACIENTES VÍTIMAS DE QUEIMADURAS COM USO DO DINAMÔMETRO
}

\section{EVALUATION OF MUSCLE STRENGTH IN PATIENTS VICTIMS OF BURNS USING THE DYNAMOMETER}

\author{
Bárbara Cristina Lopes da Silva ${ }^{1}$, Bruna de Souza Silva ${ }^{2}$, Isabela Cerri Nascimento ${ }^{3}$, Isabela Nascimento \\ Voltarelli Donato ${ }^{4}$, Isabella Mazzo Miorim ${ }^{5}$, Lucas Gonçalves Torquato ${ }^{6}$, Victoria Message Fuentes ${ }^{7}$, Adriana da \\ Costa Gonçalves ${ }^{8}$
}

Submetido em: 29/09/2021

e210829

Aprovado em: 09/11/2021

https://doi.org/10.47820/recima21.v2i10.829

\begin{abstract}
RESUMO
Objetivo: Avaliar possíveis alterações na força e função de indivíduos vítimas de queimaduras com acometimento de membros superiores. Material e Métodos: Estudo transversal, em vítimas de queimaduras, maiores de 18 anos, com cicatrizes em membros superiores, tendo o membro contralateral como controle, entre junho e agosto de 2019. Aprovação no Comitê de Ética e Pesquisa no 3327638. Na coleta de dados deste estudo foram utilizados o Termo de Consentimento Livre e Esclarecido, ficha de avaliação, dinamômetro digital (Lafayette), QuickDASH e Escala visual analógica (EVA). Resultados: Foram selecionadas 10 participantes, com média de 48,28 anos de idade (DP: 14,42), predomínio de mulheres (80\%). As áreas mais atingidas foram braço e antebraço (80\%), predomínio de destros (80\%). A média de tempo pós-operatório foi de 11,83 meses (DP: 7,35), tratamento fisioterapêutico em média por 7,88 meses (DP: 4,16), com queixas de dor (80\%) e prurido (50\%). Na EVA a média referente a dor foi de 3,5 (DP: 3,71). Na avaliação da força muscular (dinamômetro), foi observada uma diferença significativa para flexão de cotovelo $(p=0,038)$, extensão de cotovelo $(p=0,031)$ e abdução de ombro $(p=0,024)$, quando comparado o membro superior queimado com o lado contralateral, não acometido. No QuickDASH a média das notas foi de 35,68 (DP: 23,17), boa funcionalidade. Considerações finais: Indivíduos vítimas de queimaduras com acometimento unilateral de membros superiores, mesmo em uma fase tardia, apresentam alterações na força muscular em movimentos específicos quando comparados ao membro não acometido, após avaliação com dinamômetro digital, porém sua função foi classificada como boa, com a utilização do questionário QuickDASH.
\end{abstract}

PALAVRAS-CHAVE: Avaliação. Dinamômetro de força muscular. Queimadura. Membro superior. Fisioterapia.

\section{ABSTRACT}

Objective: to evaluate possible changes in strength and function in selected burns with involvement of upper limbs. Material and Methods: Cross-sectional study on death from burns, over 18 years old, with scars on the upper limbs, with the contralateral limb as control, between June and August 2019. Approval by the Ethics and Research Committee No. 3327638. In the collection of data from this study were used the Informed Consent Form, evaluation form, digital dynamometer (Lafayette), QuickDASH and visual analogue scale (VAS). Results: 10 participants were selected, with a mean age of 48.28

\footnotetext{
${ }^{1}$ Graduada em Fisioterapia pelo Centro Universitário Barão de Mauá, Ribeirão Preto-SP

2 Graduada em Fisioterapia pelo Centro Universitário Barão de Mauá, Ribeirão Preto-SP

${ }^{3}$ Graduada em Fisioterapia pelo Centro Universitário Barão de Mauá, Ribeirão Preto-SP

${ }^{4}$ Graduada em Fisioterapia pelo Centro Universitário Barão de Mauá, Ribeirão Preto-SP

5 Graduanda da Faculdade de Medicina de Catanduva (FAMECA)

${ }^{6}$ Graduando da Faculdade de Medicina de Catanduva (FAMECA)

7 Graduanda em Fisioterapia pelo Centro Universitário Barão de Mauá, Ribeirão Preto-SP

8 Doutora e Mestre pela Universidade de São Paulo (USP) e docente do curso de Fisioterapia do Centro Universitário Barão de Mauá - CBM, Ribeirão Preto-SP
} 


\section{RECIMA21 - REVISTA CIENTÍFICA MULTIDISCIPLINAR ISSN 2675-6218}

AVALIAC̄̃̃ DA FORÇA MUSCULAR EM PACIENTES VÍTIMAS DE QUEIMADURAS COM USO DO DINAMÔMETRO Bárbara Cristina Lopes da Silva, Bruna de Souza Silva, Isabela Cerri Nascimento, Isabela Nascimento Voltarelli Donato, Isabella Mazzo Miorim, Lucas Gonçalves Torquato, Victoria Message Fuentes, Adriana da Costa Gonçalves

years (SD: 14.42), with a predominance of women (80\%). The most affected areas were the arm and forearm (80\%), with a predominance of right-handers (80\%). The mean postoperative time was 11.83 months (SD: 7.35), physical therapy treatment on average for 7.88 months (SD: 4.16), with complaints of pain (80\%) and itching (50\%). In VAS, the mean for pain was 3.5 (SD: 3.71). In the assessment of muscle strength (dynamometer), a reduced difference was observed for elbow flexion $(p=0.038)$, elbow extension $(p=0.031)$ and shoulder abduction $(p=0.024)$, when comparing the burned upper limb with the contralateral side, not affected. In QuickDASH the average of grades was 35.68 (SD: 23.17), good functionality. Final considerations: Kill individuals from burns with unilateral involvement of the upper limbs, even at a late stage, show changes in muscle strength in specific movements when compared to the unaffected limb, after evaluation with a digital dynamometer, but its function was considered good, with the use of the QuickDASH questionnaire.

KEYWORDS: Evaluation. Muscle strength dynamometer. Burn. Upper Limbs. Physiotherapy.

\section{INTRODUÇÃO}

As queimaduras vêm sendo um grande problema de saúde pública, não só quanto à gravidade de suas lesões e ao grande número de complicações, mas também quanto às sequelas relevantes que marcam o paciente queimado (LIMA JÚNIOR; SERRA, 2004). As causas mais frequentes das queimaduras são a chama de fogo, escaldadura com água fervente ou outros líquidos quentes e o contato com objetos aquecidos e menos comuns são as queimaduras provocadas por choque elétrico (VALE, 2005).

A lesão por queimadura ocorre pela destruição da integridade capilar e vascular, e o comprometimento do tecido vai depender da intensidade da exposição térmica, das características da área queimada e das reações locais e sistêmicas (HELM; FISHER; CROMES JUNIOR, 2002).

As queimaduras são classificadas de acordo com a extensão e a profundidade da lesão (RIVITTI, 2018). Queimaduras de primeiro grau duram em torno de 48 a 72 horas, sem comprometimento hemodinâmico, com lesão apenas da epiderme, apresentando vermelhidão local, ardência, inchaço e calor na região. A queimadura de segundo grau, atinge a epiderme e o terço superior da derme, evoluindo com a formação de bolhas dolorosas e sua resolução se dá em torno de 14 dias. Já a queimadura de terceiro grau, acomete todas as camadas da pele e, em muitos casos, tecidos como subcutâneo, músculos e ossos (Figura 1A e 1B). Apresentam um aspecto esbranquiçado ou marmóreo com redução da elasticidade tecidual, se tornando assim rígida e pode apresentar por transparência, vasos sanguíneos trombosados, com necessidade de enxertia de pele e evolução de possíveis sequelas (LIMA JÚNIOR; SERRA, 2004).

As alterações fisiopatológicas decorrentes das queimaduras profundas são inicialmente sistêmicas. A instabilidade hemodinâmica que é desencadeada pela perda da integridade capilar, promove o deslocamento de líquidos do espaço intravascular para o espaço intersticial. Devido à má distribuição de líquidos, resultante da perda capilar, o paciente apresentará edema sistêmico maciço, principalmente nas 24 horas após a lesão, estando totalmente resolvido em 7 a 10 dias após a queimadura, por meio de reposição volêmica adequada (CANELA et al., 2011). 


\section{RECIMA21 - REVISTA CIENTÍFICA MULTIDISCIPLINAR ISSN 2675-6218}

AVALIAC̄̃̃ DA FORÇA MUSCULAR EM PACIENTES VITIMAS DE QUEIMADURAS COM USO DO DINAMÔMETRO Bárbara Cristina Lopes da Silva, Bruna de Souza Silva, Isabela Cerri Nascimento, Isabela Nascimento Voltarelli Donato, Isabella Mazzo Miorim, Lucas Gonçalves Torquato, Victoria Message Fuentes, Adriana da Costa Gonçalves

Figura 1. Queimadura com acometimento de membro superior, braço e antebraço (A) e palma da mão (B).

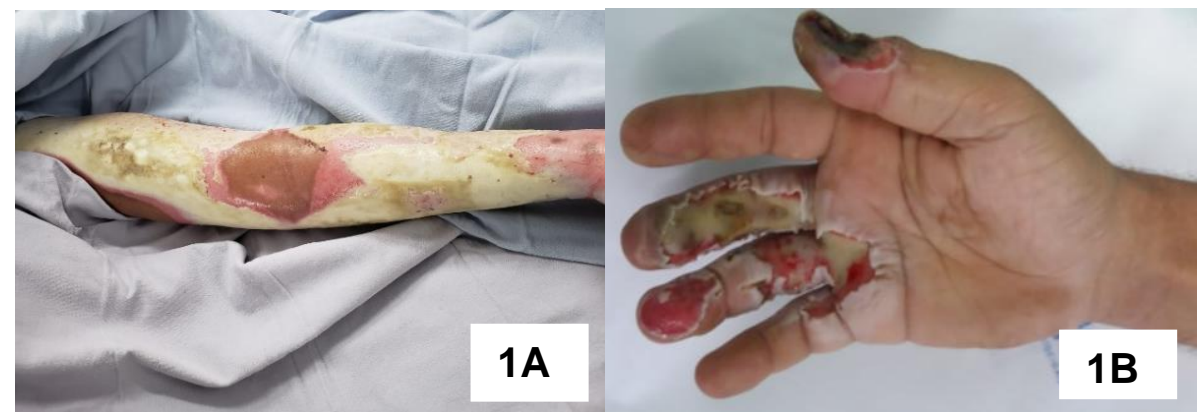

Fonte: Arquivo pessoal

Queimaduras graves geralmente são seguidas por uma resposta hipermetabólica caracterizada por aumento da temperatura corporal, aumento do consumo de glicose e oxigênio, aumento da formação de $\mathrm{CO}_{2}$, glicogenólise, lipólise e proteólise. O queimado tem seu metabolismo aumentado, assim como, catabolismo exagerado de proteína e excreção aumentada de nitrogênio urinário, ocorrendo, também, perda de proteínas pelo exsudato das feridas. Pacientes queimados são extremamente suscetíveis a infecções, o que acentua a necessidade de energia e proteína, já que após a queimadura, a proteína é catabolizada, o que leva a uma perda de massa corporal magra (Figura 2), ou seja, perda de massa muscular (LIMA; LIMA VERDE; LIMA FILHO, 2006; VINHA; CUNHA, 2007).

Figura 2. Emagrecimento evidente de paciente queimado demonstrado no momento da admissão hospitalar (A) e após 14 dias (B).
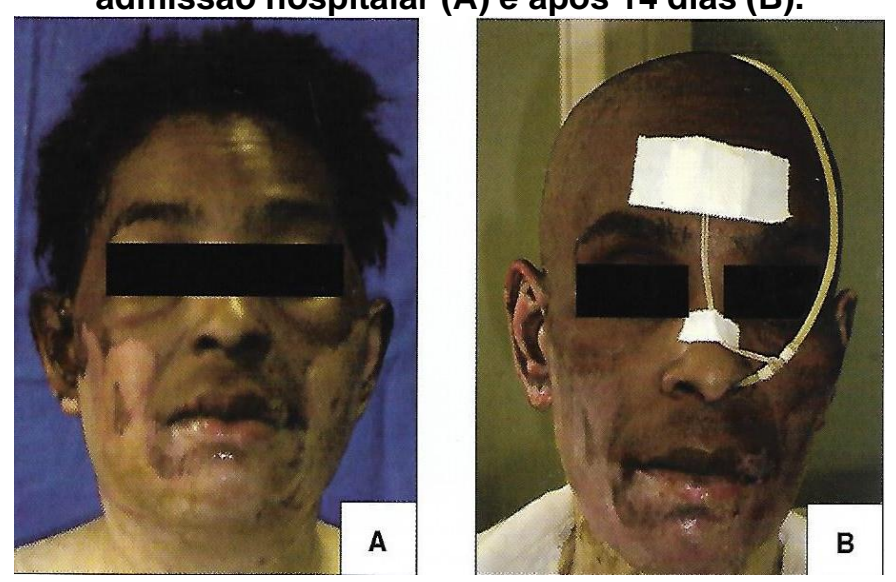

Fonte: VINHA; CUNHA, 2007.

Desta forma, o indivíduo vítima de queimaduras, pode apresentar diversas sequelas como redução da função muscular, alterações de sensibilidade local, dor, preocupação estética, problemas emocionais e de retorno às atividades de vida diárias (RYAN et al., 2015). 


\section{RECIMA21 - REVISTA CIENTÍFICA MULTIDISCIPLINAR ISSN 2675-6218}

AVALIAÇ̃̃O DA FORÇA MUSCULAR EM PACIENTES VITIMAS DE QUEIMADURAS COM USO DO DINAMÔMETRO Bárbara Cristina Lopes da Silva, Bruna de Souza Silva, Isabela Cerri Nascimento, Isabela Nascimento Voltarelli Donato, Isabella Mazzo Miorim, Lucas Gonçalves Torquato, Victoria Message Fuentes, Adriana da Costa Gonçalves

A diminuição da função muscular é um comprometimento significativo em pacientes queimados, e, para avaliar esta função, a utilização do dinamômetro isométrico portátil manual é indicado, já que o mesmo avalia força muscular de maneira confiável, com vantagens de menor custo, maior eficiência de tempo, maior portabilidade e facilidade de uso (ROMERO-FRANCO et al., 2019).

Para avaliação de incapacidades de membros superiores existem questionários específicos, dentre eles uma versão mais simplificada do Disabilities of the Arm, Shoulder and Hand (DASH), o QuickDASH, que demonstrou validade, confiabilidade e sensibilidade, detectando mudanças na função do membro superior (ATROSHI et al., 2000; BEATON; WRIGHT; KATZ, 2005; GUIMMESSON; WARD; ATROSHI, 2006). Esse questionário é composto por onze itens abordando sintomas e habilidades da vida diária em pessoas com distúrbios envolvendo o membro superior, possuindo como vantagem a mesma qualidade de informação com menos itens, facilitando sua aplicação (BEATON; WRIGHT; KATZ, 2005), tendo sido traduzido e validado para língua portuguesa por Bená (2015).

As escalas visuais analógicas (EVA) para avaliar intensidade da dor são instrumentos de medida psicométricos projetados para documentar as características da gravidade dos sintomas relacionados à doença em pacientes e usadas para obter uma classificação rápida da gravidade dos sintomas e controle da doença. A EVA também pode ser usada na rotina do paciente e para monitorar o curso de uma doença crônica (KLIMEK et al., 2017).

Dessa forma, a avaliação de vítima de queimaduras com o uso de equipamentos e escalas validadas e confiáveis se mostra necessária para mensuração das possíveis alterações e sequelas, podendo direcionar seu processo de reabilitação.

Assim, o objetivo desse estudo foi avaliar possíveis alterações na força e função de indivíduos vítimas de queimaduras com acometimento de membros superiores, utilizando dinamômetro isométrico portátil digital e a escala QuickDASH.

\section{MATERIAL E MÉTODOS}

Trata-se de um estudo transversal com abordagem quantitativa, realizado no período de junho a agosto de 2019, no qual foram convidados a participar do estudo voluntários com idade superior a 18 anos, frequentadores da Clínica de Fisioterapia Barão de Mauá, com a autorização da diretora clínica responsável, Ft. Cristiane Bernadochi D’Orsi.

Os participantes estavam de acordo em participar do estudo mediante a assinatura do Termo de Consentimento Livre e Esclarecido (TCLE). O estudo foi encaminhado ao Comitê de Ética em Pesquisa do Centro Universitário Barão de Mauá de Ribeirão Preto e aprovado com o número de protocolo 3327638.

Os critérios de inclusão do estudo foram indivíduos com idade maior de 18 anos, com cicatrizes decorrentes de queimadura de diferentes graus e acometimento de membro superior, tendo o membro superior contralateral como controle (não queimado). 


\section{RECIMA21 - REVISTA CIENTÍFICA MULTIDISCIPLINAR ISSN 2675-6218}

AVALIAÇÃO DA FORÇA MUSCULAR EM PACIENTES VÍTIMAS DE QUEIMADURAS COM USO DO DINAMÔMETRO Bárbara Cristina Lopes da Silva, Bruna de Souza Silva, Isabela Cerri Nascimento, Isabela Nascimento Voltarelli Donato, Isabella Mazzo Miorim, Lucas Gonçalves Torquato, Victoria Message Fuentes, Adriana da Costa Gonçalves

Foram excluídos deste estudo voluntários com doenças, deformidades, complicações e/ou limitações (físicas, pessoais, sociais), que pudessem interferir na avaliação, coleta de dados e procedimentos, além da recusa de assinar o TCLE.

Inicialmente, foi realizado um contato por telefone com os pacientes que se encaixavam nos critérios de inclusão e convite formal para participação do estudo. Todos os participantes do estudo tiveram esclarecimento sobre as avaliações e após a concordância e assinatura do TCLE, os pacientes foram submetidos a uma avaliação na Clínica de Fisioterapia Barão de Mauá, em uma única sessão, sendo realizada avaliação no membro superior com queimadura e no contralateral não queimado.

O presente estudo poderia causar desconfortos ou algum incômodo para responder às questões dos questionários e avaliação com os equipamentos, em relação ao tempo despendido. Os benefícios que poderão ser obtidos pela realização deste trabalho são melhora da avaliação do segmento que apresenta a cicatriz por queimaduras.

A estatística descritiva foi realizada por meio de distribuição de frequência absoluta e relativa, para as variáveis qualitativas e valor mínimo, valor máximo, média e desvio-padrão, para as variáveis quantitativas. Para comparar os lados controle e cicatriz, foi utilizado o teste paramétrico $t$-Student pareado bicaudal.

Neste estudo, foram utilizados uma ficha de avaliação, o dinamômetro isométrico portátil digital (Lafayette), QuickDASH (BENÁ, 2015), escala visual analógica (EVA) de intensidade da dor (BOTTEGA; FONTANA, 2010). A ficha de avaliação foi desenvolvida especialmente para este estudo, na qual se coletou dados pessoais (nome e telefone), dados específicos do diagnóstico e procedimentos referentes à queimadura (data do trauma, tipo de acidente, agente etiológico, grau de queimadura, cirurgia realizada, tempo pós-operatório, áreas atingidas, membro superior dominante), amplitude de movimento, limitações funcionais, força muscular, entre outros dados. Também foi realizada na avaliação a anamnese e coleta da queixa principal do voluntário.

O dinamômetro isométrico portátil digital Lafayette foi utilizado com o objetivo de mensurar o grau de força muscular do membro superior acometido por meio de uma contração isométrica máxima contra uma resistência (WESTRICK et al., 2012). Consiste em um procedimento simples (REIS; ARANTES, 2011), objetivo, prático e de fácil utilização, recomendado pela Sociedade Americana de Terapeutas de Mão (SATM). A avaliação foi realizada com o Hand-Held Dynamometer Lafayette Instrument Co. (Lafayette, IN, Model 0116, figura 3A), no membro superior acometido pela queimadura, sendo realizadas três medidas da força muscular isométrica, para posterior cálculo da média dos valores. O mesmo procedimento foi realizado no lado contralateral, não queimado. As medidas de força incluíram: flexão e abdução do ombro, flexão e extensão do cotovelo e força de preensão da mão, estando o dinamômetro isométrico portátil, posicionado na região distal do úmero, distal de antebraço e palma de mão, respectivamente (figura 3B, 3C, 3D, 3E e 3F).

As medidas foram realizadas com o participante sentado, exceto de cotovelo, na qual o mesmo ficou em decúbito dorsal (HISLOP; MONTGOMERY, 1996). Os participantes foram solicitados 


\section{RECIMA21 - REVISTA CIENTÍFICA MULTIDISCIPLINAR ISSN 2675-6218}

AVALIAC̄̃̃ DA FORÇA MUSCULAR EM PACIENTES VÍTIMAS DE QUEIMADURAS COM USO DO DINAMÔMETRO Bárbara Cristina Lopes da Silva, Bruna de Souza Silva, Isabela Cerri Nascimento, Isabela Nascimento Voltarelli Donato, Isabella Mazzo Miorim, Lucas Gonçalves Torquato, Victoria Message Fuentes, Adriana da Costa Gonçalves

a produzir uma contração máxima até o aparelho emitir um sinal sonoro (regulado para três segundos). Durante a contração, o terapeuta emitiu estímulos verbais para o paciente tais como: "Força, força, força", "Mais, mais, mais, mais", "Mais força, mais força". Ao término, era dado um reforço positivo "muito bom", "ótima tentativa". Três tentativas foram concluídas para cada lado em cada posição (WESTRICK et al., 2012), com intervalo de um minuto entre as medidas.

O QuickDASH consiste em 11 itens originais do questionário DASH sobre os sintomas e as habilidades para realizar as tarefas da vida diária durante a semana anterior à aplicação. Cada item apresenta cinco opções de resposta e os escores de todos os itens são utilizados para calcular a pontuação na escala, variando de 0 (sem deficiência) a 100 (deficiência mais grave), identificando desordens nos membros superiores (BENÁ, 2015), sendo que quanto maior a média obtida maior a disfunção do membro superior.

A Escala Visual Analógica foi utilizada com o objetivo de classificar a intensidade da dor do paciente. Esta escala é composta por uma linha horizontal com numeração de zero a dez com as extremidades indicando "ausência de dor" e "pior dor possível". O paciente classificará sua dor e ela será quantificada de acordo com o número escolhido pelo paciente (BOTTEGA; FONTANA, 2010).

Figura 3 - Hand-Held Dynamometer Lafayette Instrument Co. (Lafayette, IN, Model 01165) (3A), avaliação da preensão palmar (3B), flexão do ombro (3C), abdução do ombro (3D), flexão do cotovelo (3E) e extensão do cotovelo (3F).
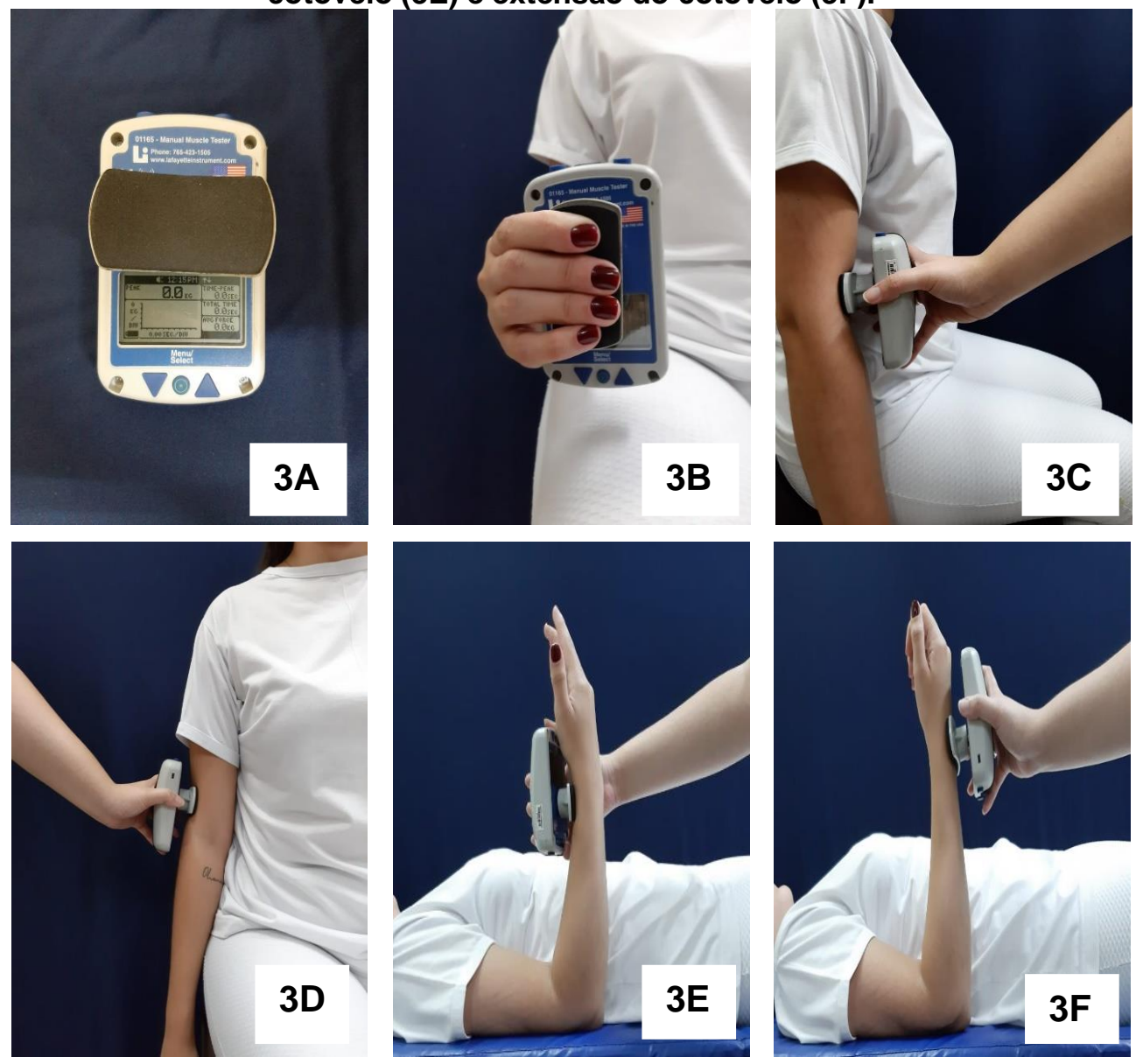

Fonte: Arquivo pessoal

RECIMA21 - Ciências Exatas e da Terra, Sociais, da Saúde, Humanas e Engenharia/Tecnologia 


\section{RECIMA21 - REVISTA CIENTÍFICA MULTIDISCIPLINAR ISSN 2675-6218}

AVALIAC̄̃̃ DA FORÇA MUSCULAR EM PACIENTES VITIMAS DE QUEIMADURAS COM USO DO DINAMÔMETRO Bárbara Cristina Lopes da Silva, Bruna de Souza Silva, Isabela Cerri Nascimento, Isabela Nascimento Voltarelli Donato, Isabella Mazzo Miorim, Lucas Gonçalves Torquato, Victoria Message Fuentes, Adriana da Costa Gonçalves

\section{RESULTADOS}

Foram avaliados 10 participantes com média de 48,28 anos de idade (DP: 14,42), com predomínio de mulheres $(80 \%, n=8)$, raça branca $(80 \%, n=8)$, maioria casados $(40 \%, n=4)$ com grau de escolaridade predominante de ensino fundamental completo $(40 \%, n=4)$ e ensino médio completo $(40 \%, n=4)$, sendo as características sociodemográficas demonstradas na Tabela 1.

Tabela 1 - Características sociodemográficas referentes às vítimas de queimaduras, dados em porcentagem (\%), média, com desvio padrão (DP).

\begin{tabular}{lr}
\hline Característica & $\%\left(\mathbf{n}^{\circ}\right.$ pacientes $)$ \\
\hline Idade & \\
Gênero & \\
Mulheres & \\
Homens & $80 \%(n=08)$ \\
Raça & $20 \%(n=02)$ \\
Branca & \\
Negra & \\
Outros & $80 \%(n=08)$ \\
& $10 \%(n=01)$ \\
\end{tabular}

\section{Estado civil}

$\begin{array}{ll}\text { Solteiro } & 20 \%(n=02) \\ \text { Casado } & 40 \%(n=04) \\ \text { Divorciado } & 10 \%(n=01) \\ \text { Viúvo } & 30 \%(n=03) \\ \text { Escolaridade } & \\ \text { Analfabeto } & \\ \text { Fundamental completo } & 00 \%(n=00) \\ \text { Fundamental incompleto } & 40 \%(n=04) \\ \text { Médio completo } & 00 \%(n=00) \\ \text { Médio incompleto } & 40 \%(n=04) \\ \end{array}$

As áreas atingidas foram os membros superiores, com predomínio de braço e antebraço na mesma porcentagem $(80 \%, n=8)$, havendo predomínio de indivíduos destros $(80 \%, n=8)$. Houve maior prevalência de queimadura de $3^{\circ}$ grau $(80 \%, n=8)$, com enxertia de pele realizada nas áreas avaliadas, com média de tempo pós-operatório de 11,83 meses (DP: 7,35 ), sendo que todos os pacientes avaliados realizaram fisioterapia em média por 7,88 meses (DP: 4,16), sendo as queixas mais comumente relatadas dor $(80 \%, n=8)$, com média da EVA de 3,5 (DP: 3,71$)$ e prurido $(50 \%$, $\mathrm{n}=5$ ). $\mathrm{O}$ tipo de acidente mais relatado foi o domiciliar em $80 \%$ dos casos, o agente causal mais frequentes o álcool em 60\%, sendo as características clínicas e cirúrgicas demonstradas na Tabela 2. 
AVALIAÇÃO DA FORÇA MUSCULAR EM PACIENTES VÍTIMAS DE QUEIMADURAS COM USO DO DINAMÔMETRO Bárbara Cristina Lopes da Silva, Bruna de Souza Silva, Isabela Cerri Nascimento, Isabela Nascimento Voltarelli Donato, Isabella Mazzo Miorim, Lucas Gonçalves Torquato, Victoria Message Fuentes, Adriana da Costa Gonçalves

Tabela 2 - Características clínicas e cirúrgicas referentes às vítimas de queimaduras, dados em porcentagem (\%).

Características $\%\left(\mathrm{n}^{\circ}\right.$ de pacientes $)$

\section{Localização da lesão}

Braço

Antebraço

Mão

\section{Lado dominante}

Direito

Esquerdo

\section{Grau de queimadura}

Segundo grau

Terceiro grau

\section{Queixas}

Dor

Estética

Prurido

Limitação funcional

Tipo de acidente

Trabalho

Domiciliar

Tentativa de suicídio

Outros

\section{Agente causal}

Álcool

Escaldadura

Outros

$$
\begin{aligned}
& 80 \%(n=08) \\
& 80 \%(n=08) \\
& 40 \%(n=04)
\end{aligned}
$$

$80 \%(n=08)$

$20 \%(n=02)$

$20 \%(n=02)$

$80 \%(n=08)$

$80 \%(\mathrm{n}=08)$

$40 \%(n=04)$

$50 \%(n=05)$

$10 \%(n=01)$

$10 \%(n=01)$

$70 \%(n=07)$

$10 \%(n=01)$

$10 \%(n=01)$

$60 \%(\mathrm{n}=06)$

$10 \%(n=01)$

$30 \%(n=03)$

Entre os indivíduos avaliados, 50\% relatavam limitação funcional e déficit de amplitude de movimento (ADM) e 60\% comprometimento da função muscular (FM), demonstrados na Tabela 3. 


\section{RECIMA21 - REVISTA CIENTÍFICA MULTIDISCIPLINAR ISSN 2675-6218}

AVALIAC̄̃̃ DA FORÇA MUSCULAR EM PACIENTES VÍTIMAS DE QUEIMADURAS COM USO DO DINAMÔMETRO Bárbara Cristina Lopes da Silva, Bruna de Souza Silva, Isabela Cerri Nascimento, Isabela Nascimento Voltarelli Donato, Isabella Mazzo Miorim, Lucas Gonçalves Torquato, Victoria Message Fuentes, Adriana da Costa Gonçalves

Tabela 3 - Valores referentes aos resultados de amplitude de movimento (ADM), limitação funcional e força muscular (FM), dados em porcentagem (\%), média, com desvio padrão (DP). Características $\%\left(n^{\circ}\right.$ de pacientes $)$

$\begin{array}{ll}\text { Limitação funcional } & \\ \text { Sim } & 50 \%(n=05) \\ \text { Não } & 50 \%(n=05) \\ \text { Limitação de ADM } & \\ \text { Sim } & 50 \%(n=05) \\ \text { Não } & 50 \%(n=05) \\ \text { Comprometimento de FM } & \\ \text { Sim } & \\ \text { Não } & 60 \%(n=06) \\ \end{array}$

Foi realizada avaliação de força muscular, utilizando o dinamômetro isométrico portátil digital (Lafayette), no membro superior com cicatriz após queimadura, comparando com o lado controle (não queimado) do indivíduo. Os movimentos avaliados foram preensão palmar, flexão e extensão de cotovelo e flexão e abdução do ombro, sendo observada uma diferença significativa para flexão de cotovelo $(p=0,038)$, extensão de cotovelo $(p=0,031)$ e abdução de ombro $(p=0,024)$, dados apresentados na figura 4 .

Figura 4 - Dados referentes a força muscular avaliada com dinamômetro isométrico portátil digital (Lafayette), comparando o lado acometido pela queimadura (cicatriz) com o lado não acometido (controle), dados em kgf.

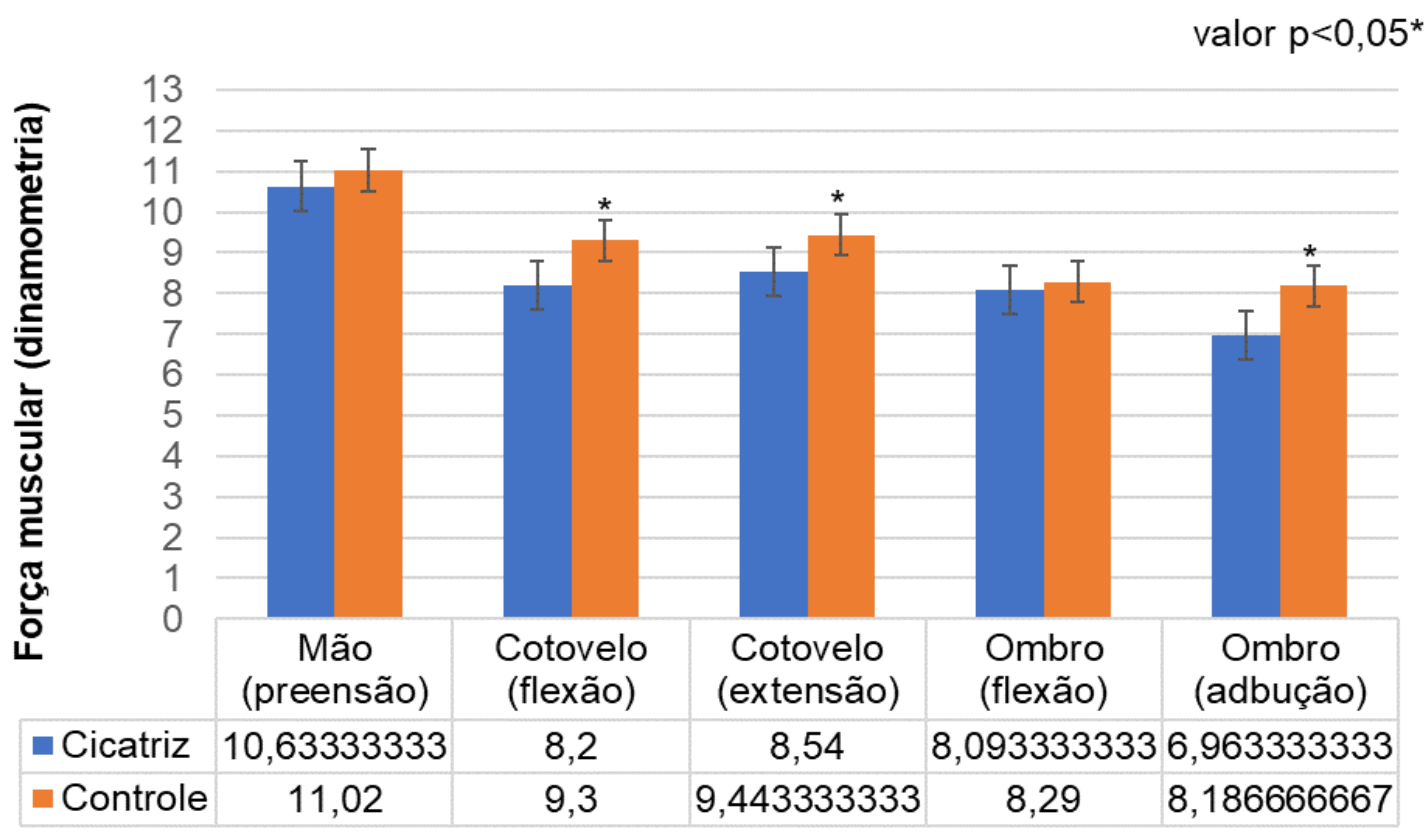




\section{RECIMA21 - REVISTA CIENTÍFICA MULTIDISCIPLINAR ISSN 2675-6218}

AVALIAÇÃO DA FORÇA MUSCULAR EM PACIENTES VÍTIMAS DE QUEIMADURAS COM USO DO DINAMÔMETRO Bárbara Cristina Lopes da Silva, Bruna de Souza Silva, Isabela Cerri Nascimento, Isabela Nascimento Voltarelli Donato, Isabella Mazzo Miorim, Lucas Gonçalves Torquato, Victoria Message Fuentes, Adriana da Costa Gonçalves

Com a utilização do questionário QuickDASH, a média encontrada em relação a disfunção de membro superior foi de 35,68 (DP: 23,17), demonstrando boa funcionalidade dos segmentos avaliados.

Ao se avaliar especificamente cada questão da escala Quick-DASH foi observado que a questão 2 (fazer tarefas domésticas pesadas), 3 (carregar uma sacola ou uma maleta), 5 (usar uma faca para cortar alimentos), 6 (atividades recreativas que exijam força ou impacto nos braços, ombros ou mãos), 7 e 8 (AVD's relacionadas a socialização e ao trabalho), tiveram as notas mais baixas variando entre 1 e 2, indicando pouca ou nenhuma dificuldade na realização destas atividades. De acordo com os resultados podemos observar que não houve uma prevalência significativa de respostas variadas entre 4 e 5, que indicam uma dificuldade extrema ou incapacidade de realizar a atividade avaliada.

\section{DISCUSSÃO}

Este estudo teve como objetivo avaliar possíveis alterações na força e função de indivíduos vítimas de queimaduras com acometimento de membros superiores e, para tanto, encontramos uma média de idade de 48,28 anos (DP: 14,42), diferentemente dos dados relatados em estudos epidemiológicos de outros autores nos quais a média de idade foi de 35,3 anos (RICCl et al., 2015), contudo, apesar das diferenças, ambos caracterizam as vítimas de queimaduras como indivíduos adultos em idade produtiva.

Com relação a outros importantes dados sociodemográficos, tanto a raça, como o sexo predominantes, fora distintos de estudos prévios. Obtivemos um predomínio da raça branca (com $80 \%$ dos casos), o que soa dissonante dos dados encontrados por outros autores em estudo realizado na cidade de Campo Grande/MS, no qual apenas 32,3\% eram brancos (UTER et al., 2012), o que pode denotar o perfil epidemiológico variável em relação à diferentes regiões. Estudos prévios sobre queimaduras demonstraram uma predominância maior no sexo masculino (LIMA; SOUSA; SANTANA, 2019; SANTOS, et al., 2017), todavia, obtivemos em nosso recrutamento um predomínio de mulheres $(80 \%)$, o que pode ser justificado pelo fato deste estudo não ter sido realizado durante o período de internação e sim, em ambiente ambulatorial.

No que concerne aos agentes causais, o álcool foi o mais frequente (em $60 \%$ dos casos), o que torna nossos dados divergentes dos relatados por outros autores (PADUA et al., 2017; ROCHA, et al., 2016), nos quais o agente causal mais prevalente foi a chama. Este fato pode ser justificado devido aos hábitos da população brasileira de acender churrasqueira utilizando o álcool, o que explica o predomínio de acidentes domiciliares, que por sinal, pode ser um ambiente inseguro caso não sejam tomadas as precauções necessárias (QUEIROZ; LIMA; ALCÂNTARA, 2013; RICCl et al., 2015).

Devido ao fato do álcool ocasionar lesões mais profundas, são indicadas cirurgias de enxertia de pele (MILCHESK; BUSNARDO; FERREIRA, 2010), que proporcionam um menor tempo de cicatrização da ferida, comumente em uma única cirurgia, permitindo reabilitação precoce do paciente

RECIMA21 - Ciências Exatas e da Terra, Sociais, da Saúde, Humanas e Engenharia/Tecnologia 


\section{RECIMA21 - REVISTA CIENTÍFICA MULTIDISCIPLINAR ISSN 2675-6218}

AVALIAÇÃO DA FORÇA MUSCULAR EM PACIENTES VÍTIMAS DE QUEIMADURAS COM USO DO DINAMÔMETRO Bárbara Cristina Lopes da Silva, Bruna de Souza Silva, Isabela Cerri Nascimento, Isabela Nascimento Voltarelli Donato, Isabella Mazzo Miorim, Lucas Gonçalves Torquato, Victoria Message Fuentes, Adriana da Costa Gonçalves

e preservando assim a função da região acometida (PAN; CHUANG; YANG, 2007). Neste estudo, os pacientes tiveram queimaduras profundas e realizaram cirurgia de enxertia de pele, apresentando boa função de membros superiores, quando avaliados com QuickDASH.

De acordo com os critérios por nós adotados, o comprometimento foi unilateral (100\%, 10), o que limitou o tamanho da amostra, sendo que, em $80 \%$ dos casos o segmento acometido foi o membro superior dominante (direito), diferente do estudo de Albuquerque et al., (2010), que relatam que a região de tórax foi a mais acometida. Por outro lado, no estudo de Batista; Martins e Schwartzman (2015) e Ricci et al., (2015), o membro superior foi o mais acometido, justificando a escolha deste trabalho de avaliar esse segmento corpóreo.

$\mathrm{Na}$ maioria dos estudos pesquisados a queixa principal relatada foi a insatisfação corporal (AIQUOC et al., 2019). Neste quesito nossos dados também são diferentes, já que $80 \%$ relataram dor de baixa intensidade, mesmo com tempo pós cirúrgicos ou de queimadura de 11,83 meses em média, provavelmente devido a queixa de prurido associada em $50 \%$ dos casos.

A diminuição de força muscular avaliada com o dinamômetro isométrico portátil Lafayette, após 11,83 meses do acidente pode ser justificada devido ao fato de pacientes vítimas de queimaduras apresentarem um alto consumo metabólico, com consequente degradação de proteínas, levando a perda de massa muscular, que ocorre sistemicamente e, que pode durar meses (SILVA et al., 2012).

Neste estudo, a perda de força muscular foi mais evidente e específica para os movimentos de flexão e extensão de cotovelo e abdução de ombro. Apesar da diminuição de força muscular, $50 \%$ dos voluntários não relatavam limitações funcionais, nem de ADM, o que pode ser justificado pelo fato de todos os pacientes terem realizados tratamento fisioterapêutico com uma média de 7,88 meses, fato que é relatado por outros autores (CIVILE; FINOTTI, 2012), nos quais são demonstrados os benefícios da fisioterapia no paciente vítima de queimaduras (ROCHA; ROCHA; SOUZA, 2010).

Vários trabalhos utilizam o dinamômetro isométrico portátil para avaliação de força em diferentes perfis de pacientes como, atletas (CAMPOS; ALVES, 2018), idosos (FERNANDES et al., 2012), mulheres portadoras de câncer de mama (VALENTE; GODOY; GODOY, 2008) e crianças (CARREIRA et al., 2010), havendo predomínio do uso do dinamômetro isométrico portátil Jamar, que avalia a força de preensão palmar especificamente.

Em nosso estudo, optamos pelo uso do dinamômetro isométrico portátil digital, para possibilitar a avaliação de diferentes movimentos e grupos musculares do membro superior. Trabalhos específicos com o dinamômetro isométrico portátil digital foram encontrados, todavia, alguns não avaliam todos os movimentos de membro superior, similar a este estudo (ROMEROFRANCO et al., 2019; WESTRICK et al., 2012).

Estudos específicos com uso de dinamômetro isométrico portátil em indivíduos vítimas de queimaduras não foram encontrados assim como os valores norteadores de normalidade em relação a dinamometria, motivo pela qual foi realizada uma avaliação comparativa com o membro 


\section{RECIMA21 - REVISTA CIENTÍFICA MULTIDISCIPLINAR ISSN 2675-6218}

AVALIAC̄̃̃ DA FORÇA MUSCULAR EM PACIENTES VITIMAS DE QUEIMADURAS COM USO DO DINAMÔMETRO Bárbara Cristina Lopes da Silva, Bruna de Souza Silva, Isabela Cerri Nascimento, Isabela Nascimento Voltarelli Donato, Isabella Mazzo Miorim, Lucas Gonçalves Torquato, Victoria Message Fuentes, Adriana da Costa Gonçalves

contralateral não queimado, sendo o indivíduo seu próprio controle, o que acabou por limitar o tamanho da amostra.

Mesmo sendo observadas alterações na força muscular com a dinamometria, em movimentos específicos, com a aplicação do QuickDASH uma boa funcionalidade, demonstrando a necessidade de utilização de instrumentos eficazes e validados que caracterizem objetivamente disfunções e otimizem o processo de avalição.

\section{CONSIDERAÇÕES FINAIS}

Indivíduos vítimas de queimaduras com acometimento unilateral de membros superiores, mesmo em uma fase tardia, apresentam alterações na força muscular em movimentos específicos quando comparados ao membro não acometido, após avaliação com dinamômetro isométrico portátil digital, porém sua função foi classificada como boa, com a utilização do questionário QuickDASH.

Avaliações específicas de funcionalidade e força podem contribuir para otimização do processo de reabilitação de indivíduos vítimas de queimaduras, inclusive com possível melhora na sua qualidade de vida.

\section{REFERÊNCIAS}

AIQUOC, K. M. et al. Avaliação da satisfação com a imagem corporal dos pacientes queimados. Revista de Enfermagem Ufpe On Line, Recife, v. 4, n. 13, p. 952-959. abr. 2019. Disponível em: https://webcache.googleusercontent.com/search?q=cache:JC4d0JKEpwMJ:https://periodicos.ufpe.br/r evistas/revistaenfermagem/article/download/237579/31773+\&cd=1\&hl=pt-BR\&ct=clnk\&gl=br. Acesso em: 30 set. 2019.

ALBUQUERQUE, M. L. L. et al. Análise dos pacientes queimados com sequelas motoras em um hospital de referência na cidade de Fortaleza-CE. Revista Brasileira de Queimaduras, Goiânia, v. 9, n. 3, p. 89-94, 28 jul. 2010. Disponível em: http://www.rbqueimaduras.com.br/details/41/pt-BR/analisedos-pacientes-queimados-com-sequelas-motoras-em-um-hospital-de-referencia-na-cidade-defortaleza-ce. Acesso em: 20 set. 2019.

ATROSHI, I. et al. Reliability and validity of the Swedish version evaluated in 176 patients. Acta Orthopaedica, [S. I.], v. 71, n. 6, p. 613-618. 2000. Disponível em: https://www.tandfonline.com/doi/abs/10.1080/000164700317362262. Acesso em: 04 abr. 2019.

BATISTA, K. T.; MARTINS, V. C. S.; SCHWARTZMAN, U. P. Y. Reabilitação em queimaduras de membros superiores. Revista Brasileira de Queimaduras, Goiânia, v. 14, n. 2, p. 114-118, 10 set. 2015. Disponível em: https://www.researchgate.net/profile/Katia Batista/publication/315772124 Perfil do paciente portado $\underline{r}$ de sequela de queimadura no membro superior atendido em Hospital de Reabilitacao/links/5 964b5a20f7e9bfb63cb6887/Perfil-do-paciente-portador-de-sequela-de-queimadura-no-membrosuperior-atendido-em-Hospital-de-Reabilitacao.pdf. Acesso em: 30 set. 2019.

BEATON, D. E.; WRIGHT, J. G.; KATZ, J. N. Development of the QuickDASH: comparison of three item-reduction approaches. Journal of bone and joint surgery, [S. I.], v. 87, p. 1038, 2005. Disponível em: ses.sp.bvs.br/lildbi/docsonline/get.php?id=5815. Acesso em: 04 abr. 2019. 


\section{RECIMA21 - REVISTA CIENTÍFICA MULTIDISCIPLINAR ISSN 2675-6218}

AVALIAÇÃO DA FORÇA MUSCULAR EM PACIENTES VÍTIMAS DE QUEIMADURAS COM USO DO DINAMÔMETRO Bárbara Cristina Lopes da Silva, Bruna de Souza Silva, Isabela Cerri Nascimento, Isabela Nascimento Voltarelli Donato, Isabella Mazzo Miorim, Lucas Gonçalves Torquato, Victoria Message Fuentes, Adriana da Costa Gonçalves

BENÁ, M. I. Validação da versão brasileira do questionário QuickDASH em pacientes portadores de sequelas de queimaduras. 2015. 28 f. Monografia (Programa de Aprimoramento Profissional/CRH/SES-SP) - FUNDAP; Hospital das Clínicas da Faculdade de Medicina de Ribeirão Preto da Universidade de São Paulo - USP, São Paulo, 2015. Disponível em: http://pesquisa.bvsalud.org/ses/resource/pt/ses-31765. Acesso em: 28 jun. 2019.

BOTTEGA, F. H.; FONTANA, R. T. A dor como quinto sinal vital: utilização da escala de avaliação por enfermeiros de um hospital geral. Scielo Analytics, Florianópolis, p. 283-290. jun. 2010. Disponível em: $\quad$ http://www.scielo.br/scielo.php?pid=S0104-07072010000200009\&script=sci abstract\&tIng=pt. Acesso em: 04 abr. 2019.

CAMPOS, M. R.; ALVES, P. H. A. Avaliação da força muscular e desempenho em teste funcional de atletas saudáveis de voleibol. 2018. 24 f. TCC (Artigo original - Graduação) - Curso de Fisioterapia, Universidade Federal de Uberlândia, Uberlândia, 2018. Disponível em: http://repositorio.ufu.br/bitstream/123456789/25701/4/Avalia\%c3\%a7\%c3\%a3oFor\%c3\%a7aMuscular. pdf. Acesso em: 30 set. 2019.

CANELA, A. F. et al. Monitorização do paciente grande queimado e as implicações na assistência de enfermagem: relato de experiência. Revista Brasileira de Queimaduras, Goiânia, v. 10, n. 4, p. 133137, 2011. Disponível em: http://www.rbqueimaduras.com.br/details/84/pt-BR/monitorizacao-dopacientegrandequeimado-e-as-implicacoes-na-assistência-de-enfermagem-relato-de-experiencia.

Acesso em: 04 abr. 2019.

CARREIRA, $\mathrm{H}$. et al. Força da preensão da mão numa amostra de crianças dos 11 aos 14 anos. Revista Científica da Ordem dos Médicos, Porto, v. 23, n. 5, p. 811-818, 30 abr. 2010. Disponível em: https://repositorio-aberto.up.pt/bitstream/10216/26092/2/47765.pdf. Acesso em: 30 set. 2019.

CIVILE, V. T.; FINOTTI, C. S. Abordagem fisioterapêutica precoce em pacientes críticos queimados. Revista Brasileira de Queimaduras, Goiânia, v. 11, n. 2, p. 85-88, 15 abr. 2012. Disponível em: http://www.rbqueimaduras.com.br/details/107/pt-BR/abordagem-fisioterapeutica-precoce-empacientes-criticos-queimados. Acesso em: 20 set. 2019.

FERNANDES, A. A. et al. Validade preditiva de equações de referência para força de preensão manual em homens brasileiros de meia idade e idosos. Fisioterapia Pesquisa, Viçosa, v. 19, n. 4, p. 351-356, set. 2012. Disponível em: http://www.scielo.br/pdf/fp/v19n4/a10v19n4. Acesso em: 30 set. 2019.

FERREIRA, E. Adaptação cultural da 'Burn Specific Health Scale-Revised' - BSHS-R: versão para brasileiros que sofreram queimaduras. 2006. $131 \mathrm{f}$. Dissertação (Mestrado) - Curso de Enfermagem, Universidade de São Paulo, Ribeirão Preto, 2006. Disponível em: http://www.teses.usp.br/teses/disponiveis/22/22132/tde-11092006-153741/pt-br.php. Acesso em: 04 abr. 2019.

GONÇALVES, N. et al. Fatores biopsicossociais que interferem na reabilitação de vítimas de queimaduras: Revisão integrativa da literatura. Revista Latino-Americana de Enfermagem, v. 19, n. 3, p. 1-9, jun. 2011. Disponível em: http://www.scielo.br/pdf/rlae/v19n3/pt 23. Acesso em: 26 nov. 2019.

GUIMMESSON, C.; WARD, M.; ATROSHI, I. The shortened disabilities of the arm, shoulder and hand questionnaire (QuickDASH): validity and reliability based on responses within the full-length DASH. BMC Musculoskeletal Disorders, v. 7, p. 44, 2006. Disponível em: https://www.ncbi.nlm.nih.gov/pmc/articles/PMC1513569/. Acesso em: 04 abr. 2019.

HELM, O. P.; FISHER, S. V.; CROMES JÚNIOR, G. F. Reabilitação de queimados. In.: DELISA, A. J.; GANS, B. M.; Tratado de medicina da reabilitação: princípios e prática. 3. ed. São Paulo: Manole, 2002. p. 1653-77. 


\section{RECIMA21 - REVISTA CIENTÍFICA MULTIDISCIPLINAR ISSN 2675-6218}

AVALIAC̄̃̃ DA FORÇA MUSCULAR EM PACIENTES VITIMAS DE QUEIMADURAS COM USO DO DINAMÔMETRO Bárbara Cristina Lopes da Silva, Bruna de Souza Silva, Isabela Cerri Nascimento, Isabela Nascimento Voltarelli Donato, Isabella Mazzo Miorim, Lucas Gonçalves Torquato, Victoria Message Fuentes, Adriana da Costa Gonçalves

HISLOP, H. J.; MONTGOMERY, J. Provas de funções muscular: técnicas de exame manual. 6. ed. Rio de Janeiro: Guanabara Koogan, 1996. 429 p.

KLIMEK, L. et al. Visual analogue scales (VAS): measuring instruments for the documentation of symptoms and therapy monitoring in cases of allergic rhinitis in everyday health care. Allergo Journal International, v. 26, n. 1, p. 16-24, 2017. Springer Nature. http://dx.doi.org/10.1007/s40629-016-0006$7 . \quad$ Disponível em: https://www.ncbi.nlm.nih.gov/pmc/articles/PMC5288410/pdf/40629 2016 Article 6.pdf. Acesso em: 04 abr. 2019.

LIMA, C. F.; SOUSA, A. R.; SANTANA, T. S. Caracterização epidemiológica de pacientes vítimas de queimaduras: realidade de um município do recôncavo baiano. Revista Saúde e Meio Ambiente, Três Lagoas, v. 1, n. 8, p. 63-74, jan. 2019. Disponível em: https://periodicos.ufms.br/index.php/sameamb/article/view/7556. Acesso em: 29 set. 2019.

LIMA JÚNIOR, E. M.; SERRA, M. C. V. F. Tratado de Queimaduras. Rio de Janeiro: Atheneu, 2004. $656 \mathrm{p}$.

LIMA, O. S.; LIMAVERDE, F. S.; LIMA FILHO, O. S. Queimados: alterações metabólicas, fisiopatologia, classificação e interseções com o tempo de jejum. In: CAVALCANTI, I. L.; CANTINHO, F. A. F.; ASSAD, A. Medicina Perioperatória. Rio de Janeiro: Sociedade de Anestesiologia do Estado do Rio de Janeiro; 2006. p. 804-815. Disponível em: http://rbqueimaduras.org.br/details/253. Acesso em: 04 abr. 2019.

MILCHESK, D. A.; BUSNARDO, F.; FERREIRA, M. C. Reconstrução microcirúrgica em queimaduras. Revista Brasileira de Queimaduras, Goiânia, v. 9, n. 3, p. 100-104, 7 ago. 2010. Disponível em: http://rbqueimaduras.com.br/details/43/pt-BR. Acesso em: 18 set. 2019.

PADUA, G. A. C. et al. Epidemiology of burn cases hospitalized at the Plastic Surgery and Burns Service of Santa Casa de Misericórdia de Santos, Brazil. Revista Brasileira de Cirurgia Plástica (rbcp) - Brazilian Journal of Plastic Sugery, v. 32, n. 4, p. 550-555, 23 set. 2017. GN1 Genesis Network. http://dx.doi.org/10.5935/2177-1235.2017rbcp0089. Disponível em: http://www.rbcp.org.br/details/1894/epidemiologia-dos-pacientes-vitimas-de-queimaduras-internadosno-servico-de-cirurgia-plastica-e-queimados-da-santa-casa-de-misericordia-de-santos. Acesso em: 20 set. 2019.

PAN, C.; CHUANG, S.; YANG, J. Thirty-eight free fasciocutaneous flap transfers in acute burned-hand injuries. Burns, Oxford, v. 33, n. 2, p. 230-235, mar. 2007. Elsevier BV. http://dx.doi.org/10.1016/j.burns.2006.06.022. Disponível em: https://www.ncbi.nlm.nih.gov/pubmed/17169493. Acesso em: 18 set. 2019.

PICCOLO, M. S. Burn Specific Health Scale-Brief: tradução para a língua portuguesa, adaptação cultural e validação. 2015. 177 f. Tese (Doutorado em Ciências) - Escola Paulista de Medicina, Universidade Federal de São Paulo, São Paulo, 2014. Disponível em: http://repositorio.unifesp.br/handle/11600/39292. Acesso em: 04 abr. 2019.

QUEIROZ, P. R.; LIMA, K. C.; ALCÂNTARA, I. C. Prevalência e fatores associados a queimaduras de terceiro grau no município de Natal, RN - Brasil. Revista Brasileira de Queimaduras, Goiânia, v. 12, n. 3, p. 169-176, ago. 2013. Disponível em: http://rbqueimaduras.org.br/details/164/pt-BR/prevalenciae-fatores-associados-a-queimaduras-de-terceiro-grau-no-municipio-de-natal--rn---brasil. Acesso em: 18 set. 2019.

REIS, M. M.; ARANTES, P. M. M. Medida da força de preensão manual - validade e confiabilidade do dinamômetro Saehan. Fisioterapia e Pesquisa, v. 18, n. 2, p. 176-181, jun. 2011. FapUNIFESP (SciELO). Disponível em: http://dx.doi.org/10.1590/s1809-29502011000200013. Acesso em: 06 jun. 2019. 


\section{RECIMA21 - REVISTA CIENTÍFICA MULTIDISCIPLINAR ISSN 2675-6218}

AVALIAÇ̃̃ DA FORÇA MUSCULAR EM PACIENTES VÍTIMAS DE QUEIMADURAS COM USO DO DINAMÔMETRO Bárbara Cristina Lopes da Silva, Bruna de Souza Silva, Isabela Cerri Nascimento, Isabela Nascimento Voltarelli Donato, Isabella Mazzo Miorim, Lucas Gonçalves Torquato, Victoria Message Fuentes, Adriana da Costa Gonçalves

RICCI, F. P. F. M. et al. Perfil epidemiológico dos pacientes com queimadura em membros superiores atendidos em uma Unidade de Queimados terciária. Revista Brasileira de Queimaduras, Goiânia, v. 14, n. 1, p. 10-13, 17 abr. 2015. Disponível em: http://rbqueimaduras.org.br/details/235/pt-BR/perfilepidemiologico-dos-pacientes-com-queimadura-em-membros-superiores-atendidos-em-uma-unidadede-queimados-terciaria. Acesso em: 18 set. 2019.

RIVITTI, E. A. Dermatologia de Sampaio e Rivitti. 4. ed. São Paulo: Artes Médicas, 2018. 1648 p.

ROCHA, J. L. F. N. et al. Qualidade de vida dos pacientes com sequelas de queimaduras atendidos no ambulatório da unidade de queimados do Hospital Regional da Asa Norte. Revista Brasileira de Queimaduras, Goiânia, v. 15, n. 1, p. 3-7, 10 jul. 2016. Disponível em: http://www.rbqueimaduras.com.br/details/286/pt-BR/qualidade-de-vida-dos-pacientes-com-sequelasde-queimaduras-atendidos-no-ambulatorio-da-unidade-de-queimados-do-hospital-regional-da-asanorte. Acesso em: 18 set. 2019.

ROCHA, M. S.; ROCHA, E. S.; SOUZA, J. P. C. Fisioterapia em queimados: uma pesquisa bibliográfica acerca dos principais recursos fisioterapêuticos e seus benefícios uma pesquisa bibliográfica acerca dos principais recursos fisioterapêuticos e seus benefícios. Revista Eletrônica de Ciências, Campina Grande, v. 9, n. 13/14, p. 01-12, jun. 2010. Disponível em: http://revistatema.facisa.edu.br/index.php/revistatema/article/view/37/55. Acesso em: 25 set. 2019.

ROMERO-FRANCO, N. et al. Validity and reliability of a low-cost dynamometer to assess maximal isometric strength of upper limb. Journal of Sports Science. v. 37, n. 15, p. 1787-1793, 2019. Disponível em: https://www.ncbi.nlm.nih.gov/pubmed/30897030. Acesso em: 30 set. 2019.

RYAN, C. M. et al. Recovery trajectories after burn injury in young adults. Journal of Burn Care \& Research, v. 36, n. 1, p. 118-129, 2015. Oxford University Press (OUP). Disponível em: http://dx.doi.org/10.1097/bcr.0000000000000214. Acesso em: 06 jun. 2019.

SANTOS, G. P. et al. Perfil epidemiológico do adulto internado em um centro de referência em tratamento de queimaduras. Revista Brasileira de Queimaduras, Goiânia, v. 16, n. 2, p. 81-86, 10 set. 2017. Disponível em: file:///C:/Users/isace/OneDrive/Área\%20de\%20Trabalho/Discussão\%20TCC/Perfil\%20epidemiológico \%20do\%20adulto\%20internado\%20em\%20um\%20centro\%20de\%20referencia\%20em\%20tratamento \%20de\%20queimados.pdf. Acesso em: 18 set. 2019.

SILVA, A. P. A. et al. Terapia nutricional em queimaduras: uma revisão. Revista Brasileira de Queimaduras, Goiânia, v. 11, n. 3, p. 135-141, ago. 2012. Disponível em: http://www.rbqueimaduras.com.br/details/119/pt-BR/terapia-nutricional-em-queimaduras--umarevisao. Acesso em: 25 set. 2019.

UTER, M. A. M. A. et al. Aspectos sociodemográficos dos pacientes tratados no setor de queimados em um hospital de alta complexidade em campo Campo Grande/MS. In: [Anais...] CONGRESSO BRASILEIRO DE QUEIMADURAS, 2012; Florianópolis: Criativa Comunicação e Editora, 2012. p.171-172.

VALE, E. Primeiro atendimento em queimaduras: a abordagem do dermatologista Inicial management of burns. Anais Brasileiros de Dermatologia: approach by dermatologists, Minas Gerais, v. 80, n. 1, p. 9-19, 11 jan. 2005. Disponível em: http://www.scielo.br/pdf/abd/v80n1/v80n01a03.pdf. Acesso em: 03 abr. 2019.

VALENTE, F. M.; GODOY, M. F. G.; GODOY, J. M. P. Força de preensão palmar em portadoras de linfedema secundário ao tratamento para câncer de mama. Arquivos de Ciência da Saúde, São José do Rio Preto, v. 15, n. 2, p. 55-58, 12 abr. 2008. Disponível em: http://repositorioracs.famerp.br/racs ol/vol-15-2/iD\%20252.pdf. Acesso em: 30 set. 2019. 


\section{RECIMA21 - REVISTA CIENTÍFICA MULTIDISCIPLINAR ISSN 2675-6218}

AVALIAÇ̃̃O DA FORÇA MUSCULAR EM PACIENTES VITIMAS DE QUEIMADURAS COM USO DO DINAMÔMETRO Bárbara Cristina Lopes da Silva, Bruna de Souza Silva, Isabela Cerri Nascimento, Isabela Nascimento Voltarelli Donato, Isabella Mazzo Miorim, Lucas Gonçalves Torquato, Victoria Message Fuentes, Adriana da Costa Gonçalves

VINHA, P. P.; CUNHA, S. F. C. Nutrição em Pacientes Queimados. In.: VANNUCCHI, H.; MARCHINI, J. S. Nutrição Clínica. Ribeirão Preto: Guanabara Koogan, 2007. Cap. 24. p. 348-354.

WESTRICK, R. B. et al. Isometric shoulder strength reference values for physically active collegiate males and females. Sports Health: a Multidisciplinary Approach, v. 5, n. 1, p. 17-21, fev. 2012. Disponível em: http://dx.doi.org/10.1177/1941738112456280. Acesso em: 20 setembro 2019.

WU, Y. et al. Evaluation of leap motion control for hand rehabilitation in burn patients: An experience in the dust explosion disaster in Formosa Fun Coast. Burns, Oxford, v. 45, n. 1, p. 157-164, fev. 2019. Disponível em: http://dx.doi.org/10.1016/i.burns.2018.08.001. Acesso em: 05 jun. 2019. 